\title{
Exploring Cognitive Playfulness Through Zero Interactions
}

\author{
Chang Hee Lee \\ Royal College of Art \\ London, SW7 2EU, UK \\ changhee.lee@network.rca.ac.uk

\section{Dan Lockton} \\ Carnegie Mellon University \\ Pittsburgh, PA 15213, USA \\ danlockton@cmu.edu \\ Ji Eun Kim \\ Royal College of Art \\ London, SW7 2EU, UK \\ jieun.kim@network.rca.ac.uk
}

Permission to make digital or hard copies of part or all of this work for personal or classroom use is granted without fee provided that copies are not made or distributed for profit or commercial advantage and that copies bear this notice and the full citation on the first page. Copyrights for third-party components of this work must be honored. For all other uses, contact the Owner/Author.

DIS'18 Companion, June 9-13, 2018, Hong Kong (C) 2018 Copyright is held by the owner/author(s). ACM ISBN 978-1-4503-5631-2/18/06.

https://doi.org/10.1145/3197391.3205413

\begin{abstract}
Many emerging technologies, products and services today try to use diverse methods of interaction to provide playful experiences. Increasingly more interactive features and techniques are being introduced to afford users new experiences and enrich our living environment. While many of these playful experiences can be achieved through various types of physical, sensory and social interactions, this paper attempts to focus on how 'no-interaction' can achieve playfulness in relation to our cognitive experience. If there is a way to give someone a playful experience without any physical, sensory and social interactions, where and how can we apply this approach or phenomenon? Here we share a provocation that tries to demonstrate a tangible means whereby such an idea could be used to explore potential user experiences within $\mathrm{HCI}$.
\end{abstract}

\section{Author Keywords}

Interaction Design; Playfulness; Cognitive Experience; Communication; Provocation.

\section{ACM Classification Keywords}

H.5.m. Information interfaces and presentation (e.g., $\mathrm{HCI}$ ): Miscellaneous. 


\section{The Playful Experiences (PLEX) Framework}

\author{
Captivation \\ Challenge \\ Competition \\ Completion \\ Control \\ Cruelty \\ Discovery \\ Eroticism \\ Exploration \\ Expression \\ Fantasy \\ Fellowship \\ Humor \\ Nurture \\ Relaxation \\ Sensation \\ Simulation \\ Submission \\ Subversion \\ Suffering \\ Sympathy \\ Thrill
}

Table 1: The Playful Experiences (PLEX) and 22 Categories [3]

\section{Introduction}

We live in what has been termed an 'experience economy', where people constantly seek and desire new kinds of experiences. Thus, establishing a positive user experience has been a central issue when designing products, services and systems $[8,12]$. This issue has been addressed in both industry and academia in terms of designing explicitly for a pleasurable experience for users [15]. One dimension of pleasurable experience can be playfulness: many services, technologies and products available today incorporate playful features.

To take disparate examples, a punch machine provides excitement by scoring a thrown punch; a dating app provides curiosity and anticipation by offering a service to find potential partners. Many of these playful components are based on interactions between users and products, accessing and manipulating people's experiences [1]; meanwhile, new approaches to experiential features are constantly being developed and introduced [2]. In this context, we decided to explore playful interactions from a rather different perspective. Instead of looking into interaction-based playfulness, we explored a form of 'cognitively' playful experience in relation to no-interaction. This study hopes to provoke unusual interactive ideas that may help to discover hidden joys for user experience.

\section{Playful Experiences}

Positive emotions such as playfulness are essential in our daily life as they provide the curiosity and capacity to learn new things [8]. Videogames offer comprehensive interactions and have been a popular form of entertainment for more than four decades [5]. Much of the research in the games industry shares different types of playful experience [9]. For example, early research introduces challenges, curiosity and fantasy as some of the essential experiences that induce a playful experience for users [11]. As such, exploring user experiences in gaming can be a useful starting point for looking at different types of playful experiences. Table 1 shows The Playful Experiences Framework (PLEX), widely known as a set of categories that helps designers make user experience more pleasurable through understanding 22 different elements of play [3]. Although the framework cannot represent all elements of play-and may never be 'complete'-categories can help us understand which aspects constitute enjoyment of interaction. In PLEX, playful experiences are largely based on integrations of physical, social and sensory interactions $[7,8]$. For example, Discovery may be achieved by exploring an uncertain territory in a game, which needs visual components and the locational property of an area; Cruelty may be achieved by causing mental or physical pain to an enemy. However, these experiences all rely on interaction with the game. Is there a way for playfulness to be developed in the mind-cognitivelywithout any actual interaction? In this paper we share a provocation that explores a way of achieving a form of cognitive playfulness, through no interaction at all.

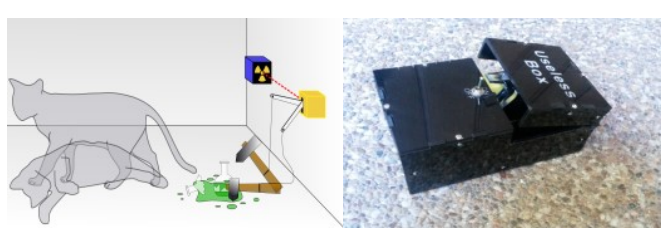

Figure 1: Inspirations - Left: Erwin Schrödinger's Imaginary Cat [16] \& Right: Marvin Minsky's Ultimate Machine [13] 'Images: Wikimedia commons' 


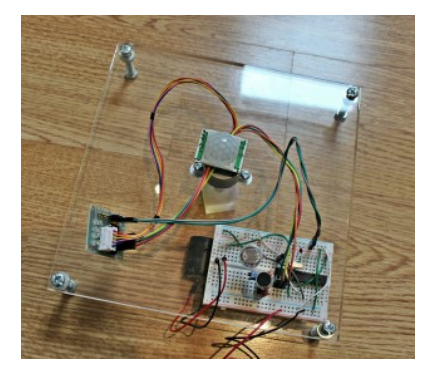

Figure 2: Silent Scene: stationary status

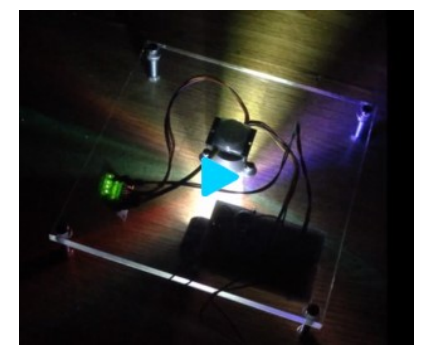

Figure 3: Silent Scene: filmed after removing sensors

(https://vimeo.com/257779013)

\section{Silent Scene}

Silent Scene is a project developed while exploring the subject of synaesthesia, a subject which deals with unusual sensation and the cognitive phenomenon of human experience [4]. However, this exploration of various unusual sensations somehow led us to explore the idea of no sensation instead of the sensation-rich experience of synaesthesia. We were also inspired by provocative precedents, such as Erwin Schrödinger's famous imaginary cat thought experiment in quantum physics (Figure 1), where he illustrates a scenario of both alive and dead as an example to explain the absurdity of the Copenhagen interpretation of quantum mechanics [6]. Another inspirational study was Marvin Minsky's 'useless machine' (Figure 1)-a device that switches off whenever someone turns it on (this can be seen as one of many novelty items that serve no practical purpose [14]). We thought some of these uncertain qualities are playful in the mind more than through actual interaction with an artefact, and they could be useful in terms of provoking debates and new kinds of experience. Therefore, we adopted uncertainty or ambiguity as one of the materials for developing a provocation centred on cognitive playfulness-

playfulness which involves thinking, knowing, believing, and so on, as its primary mechanisms.

Silent Scene (Figure 2) is an unusual machine, experiment, or device, which does not offer any interactions to anyone. Comprising ATmega328 and ATtiny 85 microcontrollers, a light head, a dichroic prism, a stepper motor and some sensors, it is a stationary device that appears to do nothing. However, when there are no humans in its environment-detected by no sound, no motion and no light-it

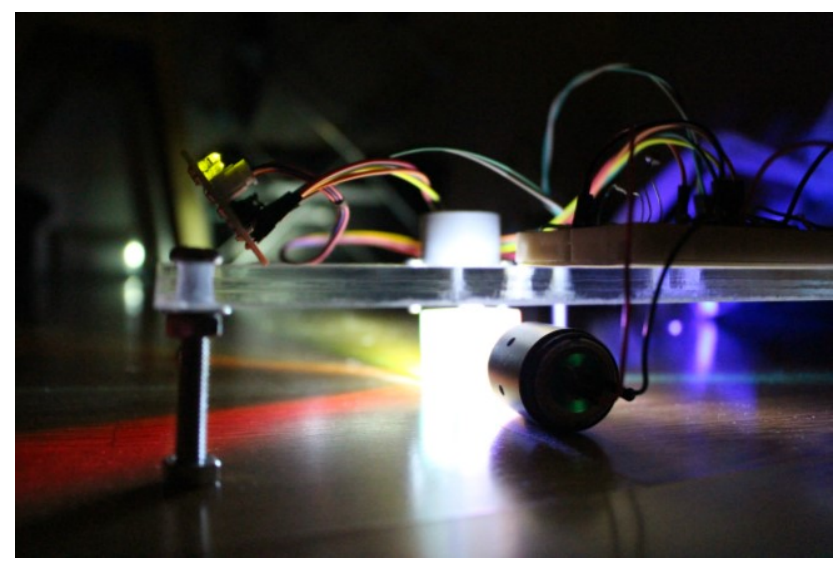

Figure 4: Silent Scene: operational status - rays of colours from prism reflection

secretly starts to create beams and rays of stunning colours, which no human can ever see (Figure $3 \& 4$ ). We considered the full spectrum of colours as a 'metaphor of total aesthetic' [10], which everyone wants to see and experience, yet no one can ever perceive. We have shared this device to a number of people in a small room, and many found the experience playful, curious and even 'joyful', although it practically provides no interaction. Below are some of the reasons they mentioned when explaining their playful experiences, primarily about cognitive processes:

Impossibility - they know something is happening beyond their interpretations, yet they are unable to experience it.

Expectation - they anticipate something is going to happen but cannot confirm it. 
Uncertainty - they are suspicious about this device and its function.

Absurdity - they have no idea what this device is trying to do.

These go beyond the categories of the PLEX Framework and offer new possibilities for cognitively playful (no)interactions. Cognitive playfulness might include a range of these kinds of factors, involving playing with thinking, knowing, believing, expecting, rationalizing, and other such cognitive processes, as prompts for designers. The implicit aim of this project is to study and discover the ways that a cognitively playful experience could occur without any direct physical, sensory and social interaction. It attempts to demonstrate this cognitive state or experience by creating a novel machine that offers zero interaction, but can generate a playful phenomenon, cognitive atmosphere and engagement.

\section{Discussion}

What we propose here is to try to discover an unusual element of play that may be helpful in shaping cognitively playful experiences for people. Especially, considering much interaction-based playfulness is being developed and introduced to the world, we thought our experiment could potentially encourage designers to envisage new types of user engagement that reinforce the playful experience. If there is a way to make someone feel playful without any interactive engagements, where and how can we employ this phenomenon to improve users' experience? What are the components that drive this type of playfulness? If there were a blue monkey hiding in your room that was impossible to encounter, would it induce a similar experience to Silent Scene? While the Silent Scene project is limited in scope, and intended primarily as a provocation, the next steps for future development of this theme may entail further exploration on different scenarios regarding how to shape and control these elements of cognitive play for potential user experiences in different services and products. This scenario's exploration and understanding may then provide a better framework that may help employing the idea of 'no-interaction' as a playful component for designing user experience.

\section{Conclusion}

The idea of 'no-interaction' in this paper was conceived while exploring the dominant environment of interaction-based playfulness, and considering the idea of cognitive playfulness as a potential addition to the dominant model. The Silent Scene project was used as a provocation to explore some possibilities of cognitive playfulness; while this paper did not articulate how to achieve this type of playful experience in detail, we have shared the provocation in the hope of developing our interest and concerns within the domain of $\mathrm{HCI}$ and design. We think this type of study hints at something that may help in designing quality experiences for users. If our interest can provoke debates around developing hidden playful interactions and techniques, this study has achieved its meaning and purpose. We hope to learn and provoke by sharing our interest and exploration to the DIS community.

\section{Acknowledgement}

We appreciate all the knowledge exchange at the Royal College of Art, Carnegie Mellon University and Harvard University. Special thanks to officer cadet Ryan S.H Han for insightful feedback. 


\section{References}

1. Monisha Akhtar. 2011. Play and Playfulness: Developmental, Cultural and Clinical Aspects. Jason Aronson, Lanham.

2. John Brotchie, Peter Newton, Peter Hall, and Peter Nijkamp. 2017. The Future of Urban Form: The Impact of New Technology. Routledge, London.

3. Brigid Costello, and Ernest Edmonds. 2007. A study in play, pleasure and interaction design. DPPI '07, 76-91.

https://dl.acm.org/citation.cfm?doid=1314161.131 4168

4. Sean A. Day. 2016. Synesthetes: A Handbook CreateSpace, North Charleston SC.

5. Isabela Granic, Adam Lobel, and Rutger C. M. E. Engels. 2013. The Benefits of Playing Video Games. American Psychologist 69, 1: 66-78.

6. Marco Genovese. 2010. Interpretations of Quantum Mechanics and the measurement problem. Advanced Science Letters, 3: 249-258. https://arxiv.org/abs/1002.0990v1

7. Wendy E. Keay-Bright. 2008. Tangible Technologies as Interactive Play Spaces for Children with Learning Difficulties: The Reactive Colours Project. The International Journal of Technology, Knowledge, and Society 4, 1: 1-10.

http://dx.doi.org/10.18848/18323669/CGP/v04i01/55821

8. Hannu Korhonen, Makrus Montola, and Juha Arrasvuori. 2009. Understanding Playful Use Experience Through Digital Games. International Conference On Designing Pleasurable Products And Interfaces, DPPI09, 274-285.

9. Andrés Lucero, Jussi Holopainen, Elina Ollila, Riku Suomela, and Evangelos Karapanos. 2013. The Playful Experiences (PLEX) Framework as a Guide for Expert Evaluation. DPPI 2013, 274-285.

https://dl.acm.org/citation.cfm?doid $=2513506.251$ 3530
10. Dan Lockton. 2018. Apophenia As Method-Or, Everything Is Either A Metaphor Or An Analogue Computer. Workshop at CHI '18.

11. Thomas Malone. 1980. What makes things fun to learn? heuristics for designing instructional computer games. SIGSMALL '80, 162-169. https://dl.acm.org/citation.cfm?doid $=800088.8028$ 39

12. Don Norman. 2005. Emotional Design: Why We Love (or Hate) Everyday Things. Basic Books, New York.

13. Wikipedia. Useless Machine. (16 May2016). Retrieved March 10, 2018 from https://en.wikipedia.org/wiki/Useless_machine\#/m edia/File:UselessMachine.png

14. Oxford English Dictionary. Novelty Item. Retrieved March 4, 2018 from

https://en.oxforddictionaries.com/definition/novelty _item

15. Kaisa Väänänen-Vainio-Mattila, and Jarmo Palviainen. 2010. Playful Experiences at Work. MobileHCI 2010, 274-285.

16. Wikipedia. Schrödinger's Cat, v.5.0. (23 February 2004). Retrieved March 7, 2018 from https://en.wikipedia.org/wiki/Schr\%C3\%B6dinger $\% 27 s \_c a t \# / m e d i a / F i l e: S c h r o d i n g e r s \_c a t . s v g$ 